\title{
Evaluación de la sensibilidad a insecticidas en poblaciones de Aedes aegypti (Diptera: Culicidae) del departamento de Caldas, Colombia, en 2007 y 2011
}

\author{
Marcela Conde', Lorena I. Orjuela', César Augusto Castellanos², Manuela Herrera-Varela', \\ Susana Licastro ${ }^{3}$, Martha L. Quiñones ${ }^{1}$ \\ 1 Grupo de Entomología, Departamento de Salud Pública, Facultad de Medicina, Universidad Nacional de \\ Colombia, Bogotá, D.C., Colombia \\ 2 Dirección Territorial de Salud de Caldas, Manizales, Colombia \\ ${ }^{3}$ Centro de Investigaciones de Plagas e Insecticidas, CIPEIN, Buenos Aires, Argentina \\ Institución donde se llevó a cabo el trabajo: \\ Universidad Nacional de Colombia, Bogotá, D.C., Colombia
}

Introducción. El uso continuo de insecticidas para el control de la transmisión del dengue puede generar una reducción en los niveles de sensibilidad de las poblaciones de los mosquitos vectores. La vigilancia oportuna es necesaria para detectar cualquier problema potencial de resistencia.

Objetivo. Determinar el estado de la sensibilidad a insecticidas de uso en salud pública en poblaciones de Aedes aegypti del departamento de Caldas, Colombia, en el 2007 y el 2011.

Materiales y métodos. Se realizaron pruebas de sensibilidad a los organofosforados temefós, malatión, fenitrotión y metil-pirimifós y al piretroide deltametrina, siguiendo las metodologías estandarizadas de la Organización Mundial de la Salud y de los Centers for Disease Control and Prevention de los Estados Unidos.

Resultados. En el 2007 se encontró resistencia al temefós en dos barrios del municipio de La Dorada, con grados de resistencia de 11,5 y 13,3. Por ello, la Dirección Territorial de Salud de Caldas suspendió el uso de este larvicida en dichos barrios. Cuatro años después, en el 2011, se observó una reducción en los grados de resistencia al temefós, así como resistencia al metil-pirimifós. Todas las poblaciones evaluadas fueron sensibles a los insecticidas deltametrina, malatión y fenitrotión en los dos períodos. Conclusión. La cuantificación de los grados de resistencia en dos períodos permitió evidenciar la reducción de la resistencia al temefós después de suspender su uso. Se recomienda mantener la vigilancia de los niveles de sensibilidad de los insecticidas usados en los programas de control de dengue.

Palabras clave: Aedes, dengue, resistencia a los insecticidas, insecticidas organofosforados, temefós, control de vectores, Colombia.

doi: http://dx.doi.org/10.7705/biomedica.v35i1.2367

Insecticide susceptibility evaluation in Aedes aegypti populations of Caldas, Colombia, in 2007 and 2011

Introduction: Continuous use of insecticides for the control of dengue transmission may lead to decreased susceptibility levels in mosquito vector populations. Timely monitoring is necessary to ensure detection of any potential resistance problems.

Objective: To determine the susceptibility status of Aedes aegypti to insecticides used in public health in Caldas, Colombia, during 2007 and 2011.

Materials and methods: Susceptibility tests to the organophosphates temephos, malathion, fenitrothion and pirimiphos methyl, as well as to the pyrethroid deltamethrin, were carried out using standard World Health Organization and Centers for Disease Control and Prevention protocols.

Results: In 2007, resistance to temephos was detected in Ae. aegypti populations from La Dorada with resistance ratios of 11.5 and 13.3, prompting the Caldas Health Department to suspend the use of this larvicide. A reduction in resistance ratios to temephos was observed in 2011, as well as an apparent resistance to pirimiphos methyl. All Ae. aegypti populations tested were susceptible to deltamethrin, malathion and fenitrothion in both years.

\section{Contribución de los autores:}

Martha L. Quiñones y César Augusto Castellanos: estructuración del proyecto

Martha L. Quiñones, Marcela Conde y Lorena I. Orjuela: trabajo de campo y laboratorio, análisis de datos y elaboración del manuscrito

Susana Licastro y Manuela Herrera-Varela: trabajo de laboratorio y análisis de datos

César Augusto Castellanos: trabajo de campo 
Conclusion: Evaluating the resistance ratios during two distinct periods allowed a decrease in resistance to be detected after suspension of the use of temephos. Surveillance of mosquito populations for changes in susceptibility levels to the insecticides used in dengue control programs is therefore recommended.

Key words: Aedes, dengue, insecticide resistance; insecticides, organophosphate; temephos, vector control, Colombia.

doi: http://dx.doi.org/10.7705/biomedica.v35i1.2367

El mosquito Aedes (Stegomyia) aegypti, Linnaeus 1762, es reconocido como el artrópodo más importante en la transmisión de los virus del género Flavivirus responsables del dengue y la fiebre amarilla urbana en humanos (1). Esta especie es también el principal vector del virus Chikungunya (Alphavirus), en torno al cual se ha generado recientemente una alerta debido a su ingreso en los países de las Américas, al incremento en el número de casos importados en la región (2) y a la presencia de casos autóctonos en países del Caribe y en algunos países de Centroamérica y Suramérica como El Salvador, Guyana y Surinam (3).

El dengue en Colombia representa un grave problema de salud pública debido a la interacción de diversas causas, entre las que se destacan el clima propicio, la urbanización no planificada, las condiciones y la calidad de vida de la población, algunos aspectos conductuales y culturales de la población, el bajo desarrollo y la respuesta institucional, la vulnerabilidad de la población expuesta, la amplia distribución del vector $A e$. aegypti, y la circulación simultánea de diferentes serotipos (4). En Colombia se reportaron entre 30.000 y 50.000 casos anuales entre el 2008 y el 2013, con excepción de la epidemia que se presentó en el 2010, cuando se registraron 147.257 casos (5). La tasa de incidencia de casos de dengue en Caldas fue de 42,8 casos por 100.000 habitantes en el 2007, y de 18,2 en el 2011, en tanto que en el año epidémico de 2010 la tasa de incidencia fue de 143,3. Los municipios de La Dorada, Victoria, Viterbo, Marquetalia y Chinchiná aportan anualmente alrededor del $70 \%$ de los casos de dengue del departamento, con incidencias en el 2011 de 164,4, 45,7, 39,5, 20,1 y 15,3 , respectivamente $(6)$.

\footnotetext{
Correspondencia:

Martha L. Quiñones, Departamento de Salud Pública, Facultad de Medicina, Avenida Carrera $30 N^{\circ}$ 45-03, Bogotá, D.C., Colombia

Teléfono: (571) 316 5000, extensión 15078

mlquinonesp@unal.edu.co

Recibido: 16/04/14; aceptado: 20/09/14
}

Debido a su amplia distribución, altas densidades, hábitos hematofágicos, cría en fuentes artificiales generadas por el hombre, y su capacidad para transmitir arbovirosis humanas, Ae. aegypti se considera una amenaza para la salud pública. A pesar de los esfuerzos globales para obtener una vacuna o un tratamiento efectivo para los pacientes con dengue $u$ otras arbovirosis transmitidas por Ae. aegypti, el control vectorial sigue siendo la única opción para la prevención y el control de estas enfermedades (1). Este control se lleva a cabo mediante la aplicación de insecticidas químicos larvicidas, como el organofosforado temefós, y adulticidas, como los organofosforados malatión, fenitrotión, metil-pirimifós y el piretroide deltametrina, que en Colombia son aplicados por funcionarios de los programas de prevención y control de enfermedades transmitidas por vectores de las secretarías de salud para reducir rápidamente las poblaciones de mosquitos y así disminuir el riesgo de transmisión del virus del dengue en situaciones epidémicas (7). Además, se ha implementado el control físico mediante la eliminación de sitios de cría, como los recipientes artificiales, o el lavado y cepillado de albercas, actividades que se desarrollan con la participación de la comunidad (7).

El organofosforado temefós ha sido el larvicida de mayor uso en los programas de control de dengue en Colombia por su efecto residual (de 8 a 12 semanas) (7), su eficacia y su costo. Como resultado de su uso frecuente, se ha reportado resistencia a este organofosforado en poblaciones de Ae. aegypti en Cali (Suárez MF, González R, Morales CA. Temephos resistance to Aedes aegypti in Cali, Colombia. Am J Trop Med Hyg. 1996;55(Suppl.):257), en Medellín (8) y en Cúcuta (9). Entre el 2005 y el 2007, se evaluó en un estudio multicéntrico el estado de la resistencia a insecticidas en los principales vectores de dengue y malaria en 12 departamentos del país y se reportó que, aproximadamente, el $70 \%$ de las poblaciones de Ae. aegypti evaluadas fueron resistentes al temefós $(10,11)$. En otros países de América Latina, como Argentina $(12,13)$, Brasil 
(14-17), Cuba $(18,19)$ y El Salvador (20), también se ha reportado la resistencia al temefós en poblaciones de Ae. aegypti.

El malatión ha sido el principal insecticida organofosforado empleado en Colombia como adulticida en casos de epidemia desde 1980 (10). En varias evaluaciones hechas en el país, se ha informado que la mayoría de las poblaciones de Ae. aegypti son sensibles al malatión $(10,11,21)$. Sin embargo, en países como Cuba, Panamá y Venezuela $(19,22,23)$, se ha encontrado una resistencia incipiente a este insecticida.

Pese a que en Colombia la deltametrina y los organofosforados fenitrotión y metil-pirimifós no se han usado de manera intensiva para el control de vectores del dengue, hay reportes de resistencia de Ae. aegypti a la deltametrina $(10,21,24)$ y al fenitrotión $(10,11,21)$. En otros países de la región, como la Guyana Francesa (25), México (26) y Perú (27), así como en el occidente venezolano (23), también se ha reportado resistencia a la deltametrina, y solo en Cuba y Panamá $(19,22,28)$, se ha encontrado resistencia entre moderada y alta al metil-pirimifós.

Teniendo en cuenta estos antecedentes, el objetivo del presente estudio fue determinar el estado de la sensibilidad de las poblaciones de Ae. aegypti a insecticidas piretroides y organofosforados en los municipios prioritarios para la transmisión del dengue, inicialmente durante el 2007. Posteriormente, en el 2011, la Dirección Territorial de Salud de Caldas seleccionó otros municipios con el fin de continuar la evaluación y sugirió incluir otro organofosforado.

En este trabajo se presentan los resultados de las evaluaciones del 2007 y del 2011, así como de las comparaciones que estas permitieron.

\section{Materiales y métodos}

\section{Área de estudio y recolección del material biológico}

Las áreas de estudio fueron seleccionadas por la Dirección Territorial de Salud de Caldas en dos periodos, el 2007 y el 2011, con base en el riesgo de transmisión del dengue y el uso de insecticidas para el control del Ae. aegypti. Las localidades donde se recolectaron los especímenes de $A e$. aegypti en el 2007 fueron los cascos urbanos de los municipios de Chinchiná (045" latitud norte, $75^{\circ} 36^{\prime \prime}$ longitud oeste), en los barrios Sector 1 y 2, cuya altitud es de 1.378 msnm, y del municipio de La Dorada $\left(05^{\circ} 27^{\prime \prime}\right.$ latitud norte, $74^{\circ} 40^{\prime \prime}$ longitud oeste), en los barrios Las Margaritas y Las Ferias, con una altitud de 176 msnm.

Dados los resultados de este primer estudio en los dos municipios, en el 2011 la Dirección Territorial de Salud de Caldas decidió cambiar el municipio de Chinchiná por otros municipios prioritarios para el control del dengue en el departamento y continuar con las evaluaciones en los mismos barrios de La Dorada. Así, en el 2011 se incluyeron los municipios de Marquetalia ( $05^{\circ} 20^{\prime \prime}$ latitud norte, $75^{\circ} 03^{\prime \prime}$ longitud oeste), con una altitud de 1.600 msnm, Victoria $\left(05^{\circ} 19^{\prime \prime}\right.$ latitud norte, $74^{\circ} 54^{\prime \prime}$ longitud oeste), con una altitud de $750 \mathrm{msnm}$, y Viterbo (0504" latitud norte, $75^{\circ} 52^{\prime \prime}$ longitud oeste), con una altitud de 998 msnm (figura 1).

Tanto en el 2007 como en el 2011 el personal de la Universidad Nacional de Colombia y los auxiliares del Programa de Enfermedades Transmitidas por Vectores de la Dirección Territorial de Salud de Caldas recolectaron larvas de Ae. aegypti en cada uno de los municipios, revisando albercas y depósitos de agua domésticos en viviendas donde los habitantes permitieran el ingreso.

Las larvas recolectadas se llevaron al Laboratorio de Entomología Médica de la Facultad de Medicina de la Universidad Nacional en Bogotá y se mantuvieron hasta la segunda generación filial (F2) para los bioensayos. Las larvas se criaron en bandejas plásticas y se les suministró concentrado para conejos como alimento. Los mosquitos adultos se alimentaron con solución de agua azucarada al $10 \%$ y con sangre humana procedente de bancos de sangre cada tercer día para la obtención de sus huevos. El insectario se mantuvo a una temperatura de $27{ }^{\circ} \mathrm{C}$ en promedio y una humedad relativa de $60 \%$, con un fotoperiodo de 12 horas, de acuerdo con la metodología de Gerberg (29).

Como referencia se utilizó la cepa sensible Rockefeller (ROCK), procedente del Laboratorio de Entomología del Instituto Nacional de Salud, la que a su vez se obtuvo en 1979 del Laboratorio de los Centers for Disease Control and Prevention (CDC) de Puerto Rico.

\section{Pruebas biológicas en larvas}

Los bioensayos con la dosis diagnóstica para temefós se ajustaron a la metodología de la Organización Mundial de la Salud (OMS) (30). Se evaluó la dosis diagnóstica de 0,02 ppm con 24 horas de exposición de las larvas. Se hicieron 


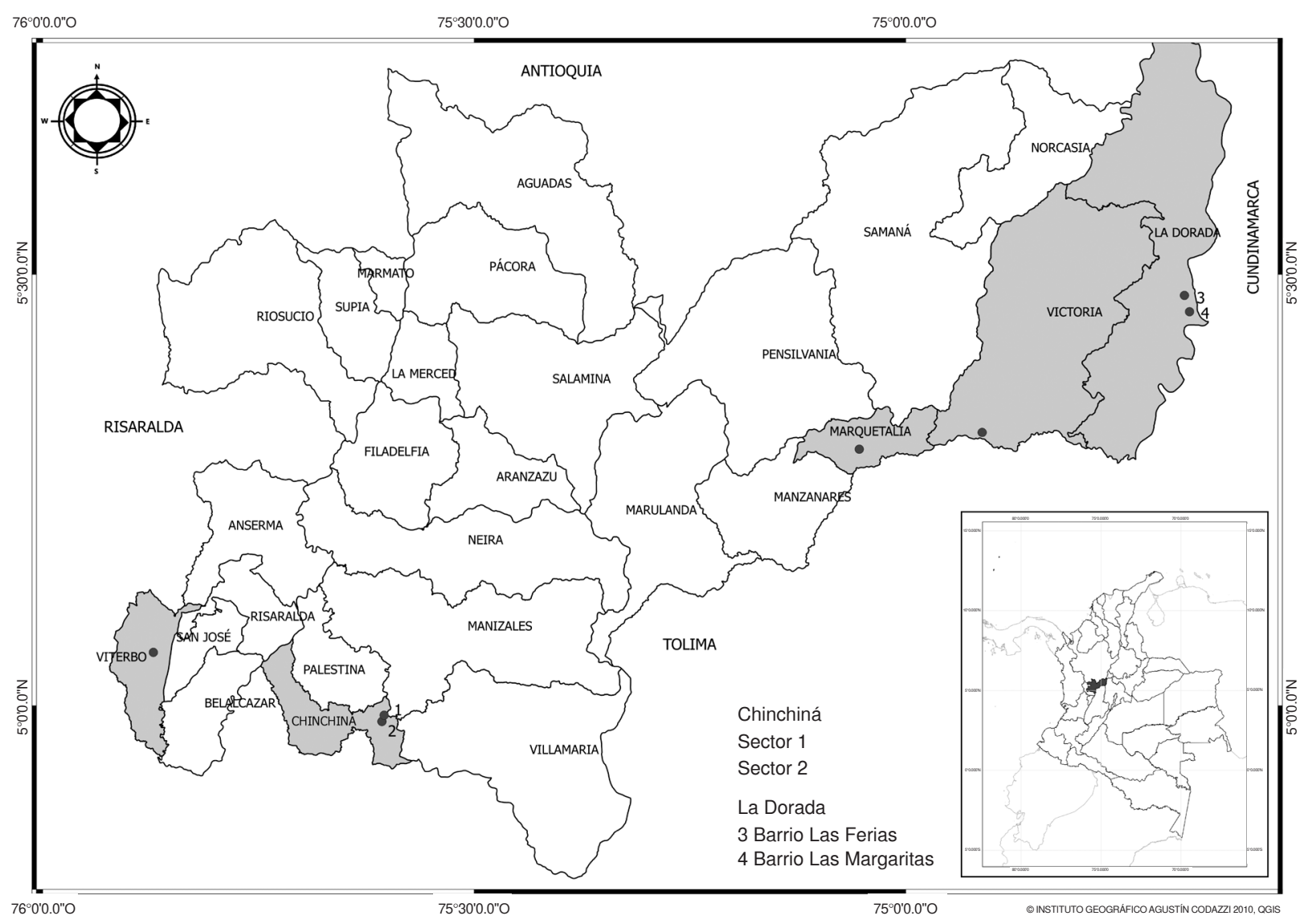

Figura 1. Ubicación de las localidades (cabeceras municipales) del departamento de Caldas en donde se recolectaron los ejemplares de Ae. aegypti para la evaluación de la resistencia a insecticidas: Chinchiná y La Dorada en el 2007 y Victoria, Viterbo, Marquetalia y La Dorada en el 2011.

Fuente cartográfica: @Instituto Geográfico Agustín Codazzi 2010. Servicio Web Feature Service. Escala 1:100.000. Límites municipales. SRC Magna Sirgas. Disponible en: http://www.igac.gov.co/wps/portal/igac/raiz/iniciohome/MapasdeColombia/Descargas.

cuatro réplicas por bioensayo, utilizando larvas de tercer estadio tardío o cuarto temprano, para un total de 100 larvas por concentración. Se utilizaron dos grupos de control: uno expuesto a la acetona (control negativo), la cual no debería causar mortalidad en las larvas, y el otro con la concentración de insecticida por evaluar en la cepa sensible ROCK (control positivo). Las larvas que pasaron a estadio de pupa durante la prueba se excluyeron del análisis, al igual que los bioensayos con mortalidades superiores al $20 \%$ en el control. La lectura de las mortalidades se hizo a las 24 horas.

Para estimar los grados de resistencia se evaluaron de 5 a 7 concentraciones que ocasionaron entre 3 y $100 \%$ de mortalidad en las larvas, tanto en las cepas de campo como en la cepa sensible de referencia ROCK. Todas las concentraciones se ajustaron con agua a un volumen final de $100 \mathrm{ml}$.

\section{Pruebas biológicas en adultos}

Para esto se utilizó la metodología de la botella del CDC descrita por Brogdon y McAllister (31). Esta metodología permite evaluar el tiempo requerido por un insecticida para alcanzar y actuar sobre el sitio de acción o sitio blanco. Este tiempo de acción se incrementa en presencia de mecanismos de resistencia.

Los insecticidas, las dosis y los tiempos diagnósticos evaluados en Ae. aegypti fueron: para deltametrina, 6,25 $\mu \mathrm{g} / \mathrm{botella} / 30$ minutos; malatión, 100 $\mu \mathrm{g} /$ botella/30 minutos; fenitrotión, $75 \mu \mathrm{g} /$ botella/45 minutos (establecidos por el proyecto multicéntrico 2005-2007) $(10,11)$, y para el metil-pirimifós, 100 ug/ botella/45 minutos, establecido por el Laboratorio de Entomología Médica de la Universidad Nacional. Las dosis diagnóstico de los insecticidas bajo evaluación se prepararon a partir de las soluciones 
madre, las cuales permanecieron protegidas de la luz y refrigeradas a $4{ }^{\circ} \mathrm{C}$. Se utilizaron soluciones de insecticida de grado analítico diluidos en acetona.

Los bioensayos se hicieron en botellas de vidrio Wheaton de $250 \mathrm{ml}$ previamente lavadas y secadas. La totalidad del interior de cada botella y de la respectiva tapa, se impregnó con una dosis diagnóstico de $1 \mathrm{ml}$ de la solución del insecticida. Esto se logró realizando movimientos de rotación hasta cerciorarse de que el producto estuviera distribuido uniformemente dentro de la botella. Las botellas se dejaron secar durante 3 a 5 horas antes de iniciar las pruebas, teniendo la precaución de mantenerlas en un sitio oscuro para proteger el insecticida de la degradación por el efecto de la luz (32).

En cada bioensayo se utilizaron cuatro botellas impregnadas con el insecticida y una botella impregnada con acetona (control negativo), en las cuales se expusieron los mosquitos de la población bajo evaluación, y en otra botella impregnada con el insecticida se expusieron mosquitos de la cepa sensible (ROCK), a manera de control positivo. En cada botella se expusieron entre 15 y 25 hembras adultas, vacías, de menos de 5 días de emergidas. Cada 15 minutos se registró el número de mosquitos vivos y muertos en cada botella. El desarrollo de la prueba tomaba de una hora y media a dos horas, o hasta que todos los mosquitos estuvieran muertos. El criterio de intoxicación en los mosquitos se definió como la dificultad para volar y la incapacidad de mantenerse parados sobre la superficie de la botella (32).

\section{Análisis de bioensayos}

Se usó la fórmula de Abbot (33) para corregir los bioensayos de larvas y adultos cuando la mortalidad en el control negativo se situaba entre 5 y $20 \%$. La interpretación de los resultados se hizo de acuerdo con los criterios de la OMS (34), los cuales establecen que mortalidades iguales 0 superiores a $98 \%$ indican sensibilidad, y aquellas inferiores a $98 \%$ indican posible resistencia.

Para los bioensayos con larvas, se estimaron las concentraciones letales al 50 y $90 \%\left(\mathrm{CL}_{50}\right.$ y $\left.\mathrm{CL}_{90}\right)$ con el insecticida temefós mediante la regresión dosis-mortalidad en el análisis de regresión Probit (SPSS $^{\circledR}, 7,5$ ). A partir de las concentraciones letales calculadas para cada cepa de Ae. aegypti, y por comparación con la línea de regresión de la cepa sensible de referencia ROCK, se calcularon los grados de resistencia para el insecticida $\left(\mathrm{CL}_{50}\right.$ de la cepa de Ae. aegypti de campo/CL $\mathrm{L}_{50}$ de la cepa ROCK). Para la interpretación de dichos grados se utilizó el criterio de Mazzarri y Georghiou (35), quienes sugieren que estos pueden clasificarse en altos (>10), medios (entre 5 y 10 ), y bajos $(<5)$.

\section{Resultados}

\section{Pruebas biológicas de la OMS con el insecticida temefós en larvas}

Las poblaciones de Ae. aegypti de Chinchiná, Marquetalia y Viterbo mostraron porcentajes de mortalidad compatibles con sensibilidad a la dosis diagnóstica (cuadro 1). Las poblaciones de los barrios de Las Ferias y Las Margaritas del municipio de

Cuadro 1. Porcentaje de mortalidad promedio y desviación estándar frente a la dosis diagnóstica de 0,02 ppm con el insecticida organofosforado temefós en ejemplares de Ae. aegypti recolectados en las localidades del departamento de Caldas en el 2007 $y$ en el 2011

\begin{tabular}{|c|c|c|c|}
\hline $\begin{array}{l}\text { Cabeceras municipales } \\
\text { y barrios }\end{array}$ & $\begin{array}{c}\text { Número de ejemplares de } \\
\text { Ae. aegypti expuestos }\end{array}$ & $\begin{array}{l}\% \text { de mortalidad promedio } \\
\pm \text { desviación estándar }\end{array}$ & Interpretación* \\
\hline \multicolumn{4}{|l|}{2007} \\
\hline \multicolumn{4}{|l|}{ La Dorada } \\
\hline Las Ferias & 295 & $45,91 \pm 14,2$ & Resistente \\
\hline Las Margaritas & 236 & $69,94 \pm 6,1$ & Resistente \\
\hline \multicolumn{4}{|l|}{ Chinchiná } \\
\hline Sector 1 & 275 & $99,6 \pm 0,5$ & Sensible \\
\hline Sector 2 & 267 & $98,53 \pm 2,5$ & Sensible \\
\hline \multicolumn{4}{|l|}{2011} \\
\hline \multicolumn{4}{|l|}{ La Dorada } \\
\hline Las Ferias & 190 & $83,5 \pm 19,09$ & Resistente \\
\hline Las Margaritas & 90 & $81,11 \pm 6,53$ & Resistente \\
\hline Victoria & 192 & $71,15 \pm 13,37$ & Resistente \\
\hline Marquetalia & 196 & $98,98 \pm 1,5$ & Sensible \\
\hline Viterbo & 100 & $99 \pm 2$ & Sensible \\
\hline
\end{tabular}

*Criterios de la Organización Mundial de la Salud, 2013 (35). 
La Dorada y la población de Ae. aegypti procedente del municipio de Victoria, mostraron porcentajes de mortalidad compatibles con resistencia.

En el cuadro 2 se presentan los grados de resistencia obtenidos para las poblaciones de Ae. aegypti de La Dorada (Las Ferias y Las Margaritas) en el 2007 y el 2011, los de Victoria, Marquetalia y Viterbo en el 2011 y los de la cepa sensible ROCK. No se incluyó la población de Chinchiná porque no se logró obtener el número mínimo de individuos requerido para esta estimación; además, los resultados con la evaluación de la dosis diagnóstica mostraron que los sectores 1 y 2 fueron compatibles con sensibilidad. Los resultados de las concentraciones letales al 50 y $90 \%$ para la cepa ROCK se estimaron en el 2007. De acuerdo con los criterios de Mazzarri y Georghiou (35), en el 2007 las poblaciones de Ae. aegypti de los barrios Las Ferias y Las Margaritas del municipio de La Dorada presentaron grados de resistencia altos, con valores de 13,27 y 11,48, respectivamente.

Durante el 2011 las poblaciones de Las Margaritas y Viterbo presentaron un grado de resistencia mayor de 5 , con valores de 5,525 y 5,61 , correspondientes a una resistencia moderada, y las poblaciones de Viterbo, Marquetalia y Las Ferias presentaron un grado de resistencia menor de 5 , con valores que oscilaron entre 0,75 y 4,75 , los cuales se consideran indicativos de sensibilidad. Las pendientes (B en el cuadro 2) de las regresiones Probit de las poblaciones de Las Margaritas en el 2007 y de Victoria en el 2011 fueron las más bajas, lo que significa que fueron más heterogéneas.

\section{Pruebas biológicas del CDC para los insecticidas deltametrina, malatión, fenitrotión y metil-pirimifós en adultos}

Los resultados de las pruebas biológicas con la metodología del CDC en mosquitos adultos se presentan en el cuadro 3. Las poblaciones evaluadas mostraron resultados compatibles con sensibilidad a todos los insecticidas evaluados tanto en el 2007 como en el 2011, a excepción de las poblaciones de Las Ferias y Las Margaritas del municipio de La Dorada, las cuales mostraron resistencia al metilpirimifós durante el 2011.

\section{Discusión}

Los resultados obtenidos con el insecticida temefós evidenciaron una reversión de la resistencia de Ae. aegypti en las poblaciones evaluadas en el departamento de Caldas. En las poblaciones de los barrios Las Ferias y Las Margaritas del municipio de La Dorada se observó una reducción en los grados de resistencia, los cuales pasaron de 13,27 y 11,48 en el 2007 a 4,75 y 5,61 durante el 2011. Esta disminución se podría explicar por la decisión de la Dirección Territorial de Salud de Caldas de suspender en estos barrios el uso del larvicida temefós a raíz de los grados de resistencia observados en el 2007. El uso de temefós se restringió solo a focos epidémicos en el departamento. Al disminuir su uso, se establecieron otras medidas de control como la educación de la comunidad, la promoción del lavado y cepillado de albercas, además de la organización de jornadas de recolección de objetos inservibles y la aplicación del tratamiento espacial con el organofosforado malatión solo en caso de epidemia, lo cual ocurrió en el 2010 (4). Esta estrategia generó una

Cuadro 2. Grados de resistencia al insecticida organofosforado temefós en Ae. aegypti de cinco localidades del departamento de Caldas en el 2007 y el 2011

\begin{tabular}{|c|c|c|c|c|c|c|c|}
\hline Localidad & $\mathrm{CL}_{50} \mathrm{ppm}$ & $\mathrm{IC}_{95 \%}$ & CL90 ppm & $I_{95 \%}$ & $\mathrm{GR} \mathrm{CL}_{50}$ & $\mathrm{GR} \mathrm{CL}_{90}$ & B \\
\hline \multicolumn{8}{|l|}{2007} \\
\hline \multicolumn{8}{|l|}{ La Dorada } \\
\hline Las Ferias & 0,031 & $0,029-0,337$ & 0,049 & $0,046-0,053$ & 13,27 & 10,59 & 4,70 \\
\hline Las Margaritas & 0,027 & $0,010-0,042$ & 0,047 & $0,036-0,112$ & 11,48 & 10,22 & 2,42 \\
\hline \multicolumn{8}{|l|}{2011} \\
\hline \multicolumn{8}{|l|}{ La Dorada } \\
\hline Las Ferias & 0,011 & $0,006-0,015$ & 0,020 & $0,016-0,033$ & 4,75 & 4,41 & 4,53 \\
\hline Las Margaritas & 0,013 & $0,010-0,016$ & 0,022 & $0,019-0,029$ & 5,61 & 4,84 & 4,54 \\
\hline Victoria & 0,013 & $0,010-0,016$ & 0,025 & $0,021-0,032$ & 5,52 & 5,49 & 2,91 \\
\hline Marquetalia & 0,006 & $0,003-0,008$ & 0,011 & $0,009-0,021$ & 2,53 & 2,51 & 3,96 \\
\hline Viterbo & 0,002 & $0,001-0,003$ & 0,003 & $0,002-0,005$ & 0,09 & 0,75 & 3,85 \\
\hline
\end{tabular}

CL: concentración letal; IC: intervalo de confianza; GR: grados de resistencia; B: valor de la pendiente de la regresión PROBIT. La cepa Rockefeller se usó como control para todas las pruebas. $\mathrm{La} \mathrm{CL}_{50}$ fue de 0,004 y el valor de la pendiente $\mathrm{B}$ fue de 4,28. Estas concentraciones se estimaron para el 2007. 
Cuadro 3. Porcentajes de mortalidad de los insecticidas deltametrina, malatión, fenitrotión y pirimifós metil con la dosis diagnóstica según las pruebas de botella del CDC en Ae. aegypti en las localidades bajo estudio en el 2007 y el 2011

\begin{tabular}{|c|c|c|c|}
\hline \multirow{2}{*}{ Insecticida } & \multirow{2}{*}{ Localidad } & \multicolumn{2}{|c|}{ Mortalidada, b } \\
\hline & & 2007 & 2011 \\
\hline \multirow[t]{7}{*}{ Deltametrina } & Chinchiná, sector 1 & $100 \pm 0,0(249)$ & - \\
\hline & Chinchiná, sector 2 & $100 \pm 0,0(334)$ & - \\
\hline & La Dorada, Las Margaritas & $100 \pm 0,0(221)$ & $99,57 \pm 0,7(292)$ \\
\hline & La Dorada, Las Ferias & $100 \pm 0,0(246)$ & $100 \pm 0,0(152)$ \\
\hline & Marquetalia & - & $100 \pm 0,0(279)$ \\
\hline & Victoria & - & $100 \pm 0,0(168)$ \\
\hline & Viterbo & - & $100 \pm 0,0(317)$ \\
\hline \multirow[t]{7}{*}{ Malatión } & Chinchiná, sector 1 & $100 \pm 0,0(229)$ & - \\
\hline & Chinchiná, sector 2 & $100 \pm 0,0(150)$ & - \\
\hline & La Dorada, Las Margaritas & $98,74 \pm 1,0(231)$ & $100 \pm 0,0(327)$ \\
\hline & La Dorada, Las Ferias & $99,63 \pm 0,6(239)$ & $97,96 \pm 0,0(277)$ \\
\hline & Marquetalia & - & $100 \pm 0,0(137)$ \\
\hline & Victoria & - & $99,48 \pm 0,7(165)$ \\
\hline & Viterbo & $100 \pm 0,0(115)$ & $100 \pm 0,0(292)$ \\
\hline \multirow{7}{*}{ Fenitrotión } & Chinchiná, sector 1 & $99,35 \pm 0,9(168)$ & - \\
\hline & Chinchiná, sector 2 & $100 \pm 0,0(204)$ & - \\
\hline & La Dorada, Las Margaritas & $100 \pm 0,0(238)$ & $99,55 \pm 0,9(455)$ \\
\hline & La Dorada, Las Ferias & - & $100 \pm 0,0(192)$ \\
\hline & Marquetalia & - & $96,95 \pm 4,3(167)$ \\
\hline & Victoria & - & $100 \pm 0,0(108)$ \\
\hline & Viterbo & $100 \pm 0,0(99)$ & $100 \pm 0,0(182)$ \\
\hline \multirow{3}{*}{ Metil pirimifós } & La Dorada, Las Margaritas & - & $93,65 \pm 9,0(163)$ \\
\hline & La Dorada, Las Ferias & - & $93,68 \pm 2,3(207)$ \\
\hline & Viterbo & - & $98(97)$ \\
\hline
\end{tabular}

a Los valores son la media de los porcentajes de mortalidad \pm desviación estándar.

${ }^{b}$ Entre paréntesis aparece el número de mosquitos evaluados.

La cepa Rockefeller se usó como control para todas las pruebas.

disminución en la presión de selección ejercida por el organofosforado temefós sobre las poblaciones de Ae. aegypti. Los resultados obtenidos con las poblaciones de Las Ferias y Las Margaritas en Caldas muestran que después de cuatro años de disminución de la presión con el temefós en poblaciones de Ae. aegypti fue posible reducir los grados de resistencia.

En los lineamientos para el manejo de la resistencia a insecticidas en vectores de enfermedades, la Organización Mundial de la Salud recomienda la rotación o aplicación en mosaico de insecticidas de diferente modo de acción, con el propósito de disminuir la presión de selección causada por un insecticida, y, eventualmente, revertir la resistencia (36). En el sector agrícola se han reportado varios casos de esta reversión, por ejemplo en el gusano del tabaco (Spodoptera litura) en Asia, en donde la sensibilidad a varios insecticidas se incrementó entre 5 y 9 veces después de 11 generaciones y en ausencia de la presión de selección (37). En el gusano cogollero (Helicoverpa armígera), plaga de varios cultivos como el algodón, se ha evidenciado la reversión de la resistencia a insecticidas con la introducción de algodón transgénico (modificado con Bacillus thurigiensis) y después de 15 años de suspensión del uso de insecticidas (38).

En el ámbito de la salud pública, en India se ha reportado una reversión de la resistencia al piretroide deltametrina en poblaciones de mosquitos de Anopheles culicifacies después de 2 a 3 años de haber suspendido su rociamiento dentro de los domicilios, aunque la misma población mostró estabilidad en la resistencia al DDT y al malatión después de haber suspendido su uso durante 30 y 9 años, respectivamente (39).

Con relación a la especie Ae. aegypti y el insecticida temefós, Melo-Santos, et al., (40) obtuvieron en Brasil una reversión de la resistencia a este organofosforado en experimentos llevados a cabo en el laboratorio con una cepa de campo resistente para evaluar tres escenarios diferentes en cuanto a la intensidad de la presión con temefós y al ingreso de individuos sensibles, y en todos ellos encontraron reversión de la resistencia, lo que demuestra que es posible recuperar la sensibilidad al temefós. En una población de Brasil (Juazeiro do 
Norte) se observó una disminución de los grados de resistencia, que pasaron de 10,2 a 7,2 después de la suspensión del uso de este organofosforado y su sustitución por Bacillus thuringiensis israelensis (41). Tales observaciones, así como los resultados de este trabajo, indican que es posible recuperar la sensibilidad al temefós, por lo cual es muy importante continuar la vigilancia del estado de la sensibilidad de las poblaciones de mosquitos a lo largo del tiempo, no solo con la dosis diagnóstica, sino estimando los grados de resistencia, lo cual permite cuantificar los niveles de resistencia y vigilar los cambios generados ya sea por el incremento o la disminución de la presión ejercida por el uso de los insecticidas (35).

Con base en los resultados de la investigación llevada a cabo en Brasil, Montella, et al., (15) mencionan la necesidad de sustituir el temefós por otro larvicida cuando las poblaciones de Ae. aegypti presenten grados de resistencia superiores a 3 , ya que con grados de resistencia superiores a 10, como los que encontraron, la eficacia en campo del temefós se disminuye a 4 semanas. Los autores concluyeron que la adopción de estas decisiones operativas requiere de una modificación enorme en la vigilancia de la resistencia que se lleva a cabo en Brasil. En este sentido, y teniendo en cuenta la ventaja de Colombia al tener programas de enfermedades transmitidas por vectores en las secretarías departamentales de salud y una Red Nacional de Laboratorios en Entomología, es importante mantener la vigilancia de la resistencia al temefós de una forma periódica y rutinaria y, en lo posible, estimar los grados de resistencia mediante los bioensayos prácticos que ha sugerido la OMS (30), y complementar esto con las pruebas bioquímicas o el uso de moléculas con sinergia, las cuales permiten detectar los mecanismos que pueden estar expresándose en las poblaciones resistentes. Además, es importante disminuir la presión ejercida por el uso del temefós, principalmente en las poblaciones de Ae. aegypti que han mostrado resultados compatibles con resistencia.

Con relación a los otros insecticidas evaluados, los resultados obtenidos en este estudio demostraron que las poblaciones de Ae. aegypti del departamento de Caldas incluidas en este estudio, fueron sensibles a la deltametrina y al fenitrotión en los dos períodos de muestreo, lo que puede deberse a que el Programa de Enfermedades Transmitidas por Vectores de Caldas no ha utilizado estos insecticidas para el control del $A e$. aegypti en ninguna de las localidades evaluadas. Sin embargo, es importante mantener la vigilancia de la sensibilidad a la deltametrina dado lo registrado en otros países de Latinoamérica con relación a una posible resistencia cruzada por presión de selección con el malatión (42), el temefós (27) y otros piretroides (24), así como con el organofosforado fenitrotión, del cual existen reportes de resistencia cruzada con el temefós (18).

Las poblaciones de Ae. aegypti de los barrios de Las Ferias y Las Margaritas del municipio de La Dorada mostraron resultados compatibles con resistencia al organofosforado metil-pirimifós, por lo que se recomienda verificarla y calcular los grados de resistencia, dado el porcentaje de mortalidad (93\%) $y$ el hecho de que en ninguna localidad de Caldas se ha empleado el metil-pirimifós. Es necesario estudiar, igualmente, una probable resistencia cruzada entre el metil-pirimifós y el temefós, ya que ha habido algunos reportes de dicha resistencia entre estos dos organofosforados en países como Cuba $(19,28,43)$ y Panamá $(19,22)$.

Con relación al malatión, las poblaciones de Ae. aegypti evaluadas fueron sensibles a este organofosforado en los dos períodos de muestreo, pese al hallazgo de poblaciones con grados de resistencia altos y moderados al temefós. Puesto que estos dos insecticidas son organofosforados, se podría pensar en el riesgo de resistencia cruzada. Sin embargo, generalmente la resistencia al malatión está relacionada con la presencia de mecanismos de resistencia diferentes a la resistencia frente a otros organofosforados, dado que las enzimas desintoxicadoras del malatión son principalmente las malatión carboxil-esterasas, las cuales actúan secuestrando el insecticida antes de que llegue a su sitio de acción (44). Es muy importante mantener la vigilancia del malatión debido a los reportes sobre resistencia cruzada entre piretroides y este organofosforado (42). Se sugiere alternar o rotar su uso con otros insecticidas en situaciones de epidemias y probar otros tipos de control en situaciones no epidémicas, como el control biológico o el físico, con el fin de disminuir la presión de selección que se ha venido generando con el uso del malatión desde hace más de tres décadas para el control del dengue en Colombia (7).

La vigilancia de los niveles de sensibilidad de las poblaciones de mosquitos vectores requiere de un seguimiento a lo largo del tiempo para clasificar las localidades que están en riesgo de resistencia incipiente, moderada o alta. Esto debería llevarse 
a cabo conjuntamente con pruebas de eficacia de los productos en campo, a fin de tomar mejores decisiones en cuanto al uso adecuado de los insecticidas y el manejo de la resistencia.

En conclusión, la determinación de los grados de resistencia en poblaciones de Ae. aegypti en Caldas permitió cuantificar la disminución de la resistencia al temefós después de la suspensión de su uso. Estos resultados resaltan la importancia de mantener la vigilancia de los grados de resistencia de Ae. aegypti al temefós dada la presión que su uso ha ejercido, y evidencian, asimismo, la posibilidad de revertir la resistencia a dicho insecticida suspendiendo su aplicación y cambiando las estrategias de control, como lo hizo la Dirección Territorial de Salud de Caldas. El conocimiento sobre la estabilidad de la resistencia y su posible reversión al disminuir la presión de selección es fundamental para el diseño de estrategias de manejo de la resistencia a los insecticidas.

\section{Agradecimientos}

A los funcionarios de la Secretaría de Salud de Caldas, por su colaboración y acompañamiento en la recolección del material. A la comunidad de Chinchiná, La Dorada, Marquetalia, Victoria y Viterbo, por abrirnos las puertas de sus casas y permitir la recolección del material. A Rubén Albarracín, por la elaboración del mapa.

\section{Conflicto de intereses}

Los autores manifestamos no tener conflictos de intereses en esta publicación.

\section{Financiación}

Este proyecto contó con la financiación de la Dirección Territorial de Salud de Caldas y la Universidad Nacional de Colombia a través de los convenios interadministrativos 00175 de 2007 y 00704 de 2009, y de Colciencias por medio del Programa de Intercambio Internacional de Investigadores e Innovadores en el marco de proyectos de investigación conjunta, 2011, código CO/04/11.

\section{Referencias}

1. Gubler DJ. Dengue and dengue hemorrhagic fever. Semin Pediatr Infect Dis. 1997;8:3-9.

2. Vega-Rúa A, Zouache K, Girod R, Failloux AB, Lourençode-Oliveira R. High vector competence of Aedes aegypti and Aedes albopictus from ten American countries as a crucial factor of the spread of Chikungunya. J Virol. 2014;88:6294306. http://dx.doi.org/10.1128/JVI.00370-14JVI.00370-14

3. Pan American Health Organization. Number of reported cases of Chikungunya fever in the Americas, by country or territory 2013-2014 (to week noted). Chikungunya fever in the Americas. Cumulative cases epidemiological week/ EW 30. Fecha de consulta: 25 de julio de 2014. Disponible en: http://www.paho.org/hq/index.php?option=com_ docman\&task=doc_download\&ltemid=270\&gid $=26869 \& \mathrm{I}$ ang=en

4. Padilla JC, Rojas DP, Sáenz-Gómez R. Dengue en Colombia: epidemiologia de la reemergencia a la hiperendemia. Bogotá: Guías de Impresión Ltda.; 2012. 250 p.

5. Ministerio de Salud. Informe situación del dengue. Fecha de consulta: 3 de junio de 2014. Disponible en: http://www. minsalud.gov.co/Documentos\%20y\%20Publicaciones/ INFORME\%20SITUACION\%20DE\%20DENGUE.pdf.

6. Dirección Territorial de Salud de Caldas. Informe comportamiento del dengue en el departamento de Caldas 20072012. Fecha de consulta: 11 de marzo de 2014. Disponible en: http://190.26.195.115/saludpublica/An\%C3\%A1lisis\%20 por\%20evento\%20de\%20inter\%C3\%A9s\%20en\%20 salud\%20p\%C3\%BAblica\%202007-\%202012/DENGUE.pdf.

7. Ministerio de la Protección Social, Instituto Nacional de Salud, Organización Panamericana de la Salud. Gestión para la vigilancia entomológica y control de la transmisión del dengue. Fecha de consulta: 26 de julio de 2014. Disponible en: http://www.ins.gov.co/temas-de-interes/ Dengue/03\%20Vigilancia\%20entomo\%20dengue.pdf.

8. Mazzari MB. Revisión del estado actual de la resistencia en Aedes aegypti a insecticidas utilizados en salud pública. Bol Dir Malariol Saneam Ambient 1995;35:91-5.

9. Grisales N, Poupardin R, Gómez S, Fonseca-González I, Ranson H, Lenhart A. Temephos resistance in Aedes aegypti in Colombia compromises dengue vector control. PLoS Negl Trop Dis. 2013;7:e2438. http://dx.doi.org/10. 1371/journal.pntd.0002438

10. Ocampo C, Salazar M, Mina N, Mcallister J, Brogdon W. Insecticide resistance status of Aedes aegypti in 10 localities in Colombia. Acta Trop. 2011;118:37-44. http:// dx.doi.org/10.1016/j.actatropica.2011.01.007

11. Santacoloma L, Chaves B, Brochero HL. Estado de la susceptibilidad de poblaciones naturales del vector del dengue a insecticidas en trece localidades de Colombia. Biomédica. 2012;32:333-43. http://dx.doi.org/10.7705/ biomedica.v32i3.680

12. Seccacini E, Lucia A, Zerba E, Licastro S, Masuh H. Aedes aegypti resistance to temephos in Argentina. J Am Mosq Control Assoc. 2008;24:608-9.

13. Llinás GA, Seccacini E, Gardenal CN, Licastro S. Current resistance status to temephos in Aedes aegypti from different regions of Argentina. Mem Inst Oswaldo Cruz. 2010;105:113-6. http://dx.doi.org/10.1590/S007402762010000100019

14. Lima JB, Da-Cunha MP, Da Silva RC, Galardo AK, Soares Sda S, Braga IM, et al. Resistance of Aedes aegypti to organophosphates in several municipalities in the State of Rio de Janeiro and Espírito Santo, Brazil. Am J Trop Med Hyg. 2003;68:329-33.

15. Montella I, Martins A, Viana P, Lima J, Braga I, Valle D. Insecticide resistance mechanisms of Brazilian Aedes aegypti populations from 2001 to 2004. Am J Trop Med Hyg. 2007;77:467-77.

16. Araujo AP, Araujo-Diniz DF, Helvecio E, de Barros RA, de Oliveira CM, Ayres CF, et al. The susceptibility of 
Aedes aegypti populations displaying temephos resistance to Bacillus thuringiensis israelensis: A basis for management. Parasit Vectors. 2013;6:297-305. http://dx. doi.org/10.1186/1756-3305-6-297

17. Gambarra WP, Martins WF, de Lucena Filho ML, de Albuquerque IM, Apolinário OK, Beserra EB. Spatial distribution and esterase activity in populations of Aedes (Stegomyia) aegypti (Linnaeus) (Diptera: Culicidae) resistant to temephos. Rev Soc Bras Med Trop. 2013;46:178-84. http://dx.doi.org/10.1590/0037-8682-1727-2013

18. Rodríguez MM, Bisset JA, Fernández D, Pérez O. Resistencia a insecticidas en larvas y adultos de Aedes aegypti: prevalencia de la esterasa A4 asociada con la resistencia a temephos. Rev Cubana Med Trop. 2004;56:54-60.

19. Rodríguez MM, Bisset JA, Fernández D. Levels of insecticide resistance and resistance mechanisms in Aedes aegypti from some Latin American Countries. J Am Mosq Control Assoc. 2007;23:420-9.

20. Bisset JA, Rodríguez MM, San Martín JL, Romero JE, Montoya R. Evaluación de la resistencia a insecticidas de una cepa de Aedes aegypti de El Salvador. Rev Panam Salud Pública. 2009;26:229-34. http://dx.doi.org/10.1590/ S1020-49892009000900007

21. Fonseca-González I, Quiñones ML, Lenhart A, Brogdon WG. Insecticide resistance status of Aedes aegypti (L.) from Colombia. Pest Manag Sci. 2010;67:430-7. http://dx.doi. org/10.1002/ps.2081

22. Bisset JA, Rodríguez MM, Cáceres L. Niveles de resistencia a insecticidas y sus mecanismos en dos cepas de Aedes aegypti de Panamá. Rev Cubana Med Trop. 2003;55:191-5.

23. Álvarez LC, Ponce G, Oviedo M, López B, Flores AE. Resistance to malathion and deltamethrin in Aedes aegypti (Diptera: Culicidae) from western Venezuela. J Med Entomol. 2013;50:1031-9. http://dx.doi.org/10.1603/ME12254

24. Santacoloma L, Chaves B, Brochero HL. Susceptibilidad de Aedes aegypti a DDT, deltametrina y lambdacialotrina en Colombia. Rev Panam Salud Pública. 2010; 27:66-73. http://dx.doi.org/10.1590/S1020-49892010000100010

25. Dusfour I, Thalmensy V, Gaborit P, Issaly J, Carinci $\mathbf{R}$, Girod R. Multiple insecticide resistance in Aedes aegypti (Diptera: Culicidae) populations compromises the effectiveness of dengue vector control in French Guiana. Mem Inst Oswaldo Cruz. 2011;106:346-52. http://dx.doi. org/10.1590/S0074-02762011000300015

26. Flores AE, Ponce G, Silva BG, Gutiérrez SM, Bobadilla C, López B, et al. Wide spread cross resistance to pyrethroids in Aedes aegypti (Diptera: Culicidae) from Veracruz State, México. J Econ Entomol. 2013;106:959-69. http://dx.doi. org/10.1603/EC12284

27. Vargas F, Córdova O, Alvarado A. Determinación de la resistencia a insecticidas en Aedes aegypti, Anopheles albimanus y Lutzomyia peruensis procedentes del norte Peruano. Rev Peru Med Exp Salud Pública. 2006;23:259-64.

28. Bisset JA, Rodríguez MM, Molina D, Díaz C, Soca LA. Esterasas elevadas como mecanismo de resistencia a insecticidas organofosforados en cepas de Aedes aegypti. Rev Cubana Med Trop. 2001;53:37-43.

29. Gerberg EJ. Manual for mosquito rearing and experimental techniques. Am Mosq Control Assoc. 1970;5:1-9
30. World Health Organization. Instructions for determining the susceptibility or resistance of mosquito larvae to insecticides. WHO/VBC/ 81.807. Geneva: WHO; 1981. p. 6.

31. Brogdon WG, McAllister JC. Simplification of adult mosquito bioassays through use of time-mortality determinations in glass bottles. J Am Mosq Control Assoc. 1998;14:159-64.

32. Centers for Disease Control and Prevention. Guideline for evaluating insecticide resistance in vectors using the CDC bottle bioassay. First edition. Washington, D.C.: CDC; 2010. p.56.

33. Abbot AW. A method of computing the effectiveness of an insecticide. J Am Mosq Control Assoc. 1987;3:302-3.

34. World Health Organization. Test procedures for insecticide resistance monitoring in malaria vector mosquitoes. Geneva: WHO; 2013. p.30.

35. Mazarri MB, Georghiou GP. Characterization of resistance to organophosphate, carbamate, and pyrethroid insecticides in field populations of Aedes aegypti from Venezuela. J Am Mosq Control Assoc. 1995;11:315-22.

36. World Health Organization. Global plan for insecticide resistance management in malaria vectors (GPIRM). Geneva: WHO; 2012. Fecha de consulta: 19 de junio de 2014. Disponible en: http://www.who.int/malaria/vector_control/ivm/ gpirm/en/index.html.

37. Rehan A, Saleem MA, Freed S. Baseline susceptibility and stability of insecticide resistance of Spodoptera lityra (F.) (Lepidoptera:Noctuidae) in the absence of selection pressure. Pakistan J. Zool. 2011;43:973-8.

38. Yang Y, Li Y, Wu Y. Current status of insecticide resistance in Helicoverpa armigera after 15 years of Bt cotton planting in China. J Econ Entomol. 2013;106:375-81.

39. Raghavendra K, Vema V, Srivastava HC, Gunasekaran K, Sreehari U, Dash AP. Persistance of DDT, malathion \& deltamethrin resistance in Anopheles culicifascies after their sequential withdrawal from indoor residual spraying in Surat district, India. Indian J Med Res. 2010;132:260-4

40. Melo-Santos MA, Varjal-Melo JJ, Araújo AP, Gomes TC, Paiva MH, Regis LN, et al. Resistance to the organophosphate temephos: Mechanisms, evolution and reversion in an Aedes aegypti laboratory strain from Brazil. Acta Trop. 2010;113:180-9. http://dx.doi.org/10.1016/j. actatropica.2009.10.015

41. Lima EP, Paiva MH, Araujo AP, da Silva EVG, da Silva M, de Oiveiras LN, et al. Insecticide resistance in Aedes aegypti populations from Ceará, Brazil. Parasit Vectors. 2011;4:5. http://dx.doi.org/10.1186/1756-3305-4-5

42. Rodríguez MM, Bisset JA, Díaz C, Soca LA. Cross resistance to pyrethroids in Aedes aegypti from Cuba induced by the selection with organophosphate malathion. Rev Cubana Med Trop. 2003;55:105-11.

43. Rodríguez MM, Bisset JA, Milá LH, Calvo E, Díaz C, Soca LA. Niveles de resistencia a insecticidas y sus mecanismos en una cepa de Aedes aegypti de Santiago de Cuba. Rev Cubana Med Trop. 1999;51:83-8.

44. Fonseca I, Quiñones ML. Resistencia a insecticidas en mosquitos (Diptera: Culicidae): mecanismos, detección y vigilancia en salud pública. Rev Colomb Entomol. 2005;31:107-15. 